\title{
Le corps endormi au cœur du fait divers dans le monde rural du premier $\mathrm{XIX}^{\mathrm{e}}$ siècle
}

\section{Guillaume Garnier}

\section{(2) OpenEdition \\ Journals}

\section{Édition électronique}

URL : http://journals.openedition.org/abpo/143

DOI : $10.4000 / a b p o .143$

ISBN : 978-2-7535-1513-0

ISSN : 2108-6443

Éditeur

Presses universitaires de Rennes

Édition imprimée

Date de publication : 1 mars 2009

Pagination : $31-45$

ISBN : 978-2-7535-0875-0

ISSN : 0399-0826

\section{Référence électronique}

Guillaume Garnier, "Le corps endormi au cœur du fait divers dans le monde rural du premier XIX siècle », Annales de Bretagne et des Pays de l'Ouest [En ligne], 116-1 | 2009, mis en ligne le 20 mars 2011, consulté le 19 avril 2019. URL : http://journals.openedition.org/abpo/143 ; DOI : 10.4000/ abpo. 143 


\title{
Le corps endormi au cœur du fait divers dans le monde rural du premier XIX ${ }^{\mathrm{e}}$ siècle
}

\author{
Guillaume GARNIER \\ Professeur certifié d'histoire-géographie, \\ Doctorant en histoire moderne et contemporaine, \\ université de Genève et université de Poitiers
}

À la fin de l'année 1820, la petite ville de Méré ${ }^{1}$ se retrouve au cœur d'un tragique fait divers. L'affaire a débuté le 22 septembre 1820. Ce jour-là, quelques habitants de Méré et de la commune de "Barous ${ }^{2}$ " se mobilisaient pour rechercher Anne Berthelot, née Anne Martin, qui s'était apparemment noyée dans la Creuse. Jean Ribreau, pêcheur de la commune de Barrou, témoignait qu'en revenant de la foire de Lésigny, "le bonhomme Berthelot vint [le] prier d'aller chercher sa femme dans la rivière en [lui] disant qu'il avait trouvé une capote accrochée à une racine ${ }^{3}$ ». Ils y allèrent " par trois fois » sans succès. Anne Berthelot fut déclarée disparue et les espoirs de la retrouver étaient minces.

Une rencontre malheureuse, plutôt une odeur désagréable, va faire passer Joseph Berthelot de l'état de veuf éploré soutenu par l'opinion publique, à celui de menteur suspecté de meurtre. Le 11 octobre 1820, des voisins qui passaient près du puits commun sont attirés par une forte odeur. II n'en faut pas plus pour qu'ils y découvrent rapidement le corps d'Anne Berthelot en état de décomposition. Très vite, Joseph Berthelot avoue son crime : il a tué sa femme une nuit de septembre. Les langues vont alors se délier et la nouvelle se répand dans les environs. Pendant quelques mois, cette affaire locale va troubler, mais aussi intéresser la population des environs

1. Méré est une petite commune du département de la Vienne. Elle est située à une quinzaine de kilomètres à l'est de Châtellerault, au bord de la rivière de la Creuse (qui sépare d'ailleurs le département de la Vienne du département de I'Indre-et-Loire). Dans cet article, l'orthographe du XIXe siècle qui écrit "Méré » avec un "é » sera préservée. Actuellement cette commune s'appelle Mairé.

2. Barrou (orthographe actuelle) est une commune du département de l'Indre-et-Loire située à quelques kilomètres de Méré.

3. Arch. dép. de la Vienne, 2 U 542. 
de Méré et ce, pour plusieurs raisons. Tout d'abord, il est évident qu'un crime à proximité de chez eux ne peut que mobiliser l'attention des voisins et alimenter les conversations. Dans un deuxième temps, les témoins interrogés sont étonnés par le geste du criminel : ils connaissaient l'homme, les relations houleuses qu'il entretenait avec sa femme, mais ne pensaient pas qu'ils en viendraient aux mains. Ils veulent connaître la raison de son acte, voire les détails. Enfin, il faut noter que si ce genre de fait divers attise la curiosité, c'est qu'il peut aussi engendrer des craintes et faire ressortir des angoisses collectives. C'est en partie sur ce dernier point que nous allons nous attarder dans cet article. Si la passion des populations pour les faits divers n'est plus à prouver, il est par contre très intéressant de se pencher sur un des éléments qui participent à cet attrait. En l'occurrence, ce fait divers criminel qui touche Anne Berthelot va nous permettre de comprendre la fascination, mais aussi la répulsion de la société rurale poitevine ${ }^{4}$ du XIX siècle, envers leur environnement nocturne. Comme l'ont montré Pierre Mannoni et Jean Delumeau, la nuit fait ressurgir des peurs objectives ou fantasmées, et tous les groupes humains ont eu l'expérience de la nuit inquiétante ${ }^{5}$. Les Poitevins ne semblent pas y avoir échappé, comme ils goûtaient aussi aux rencontres illégitimes et au libertinage nocturnes. Mais les témoignages de faits divers ne font pas que nous renseigner sur la nuit, ils nous orientent sur une piste délaissée par les historiens, à savoir la relation des hommes à leur corps endormi ${ }^{6}$. La pression de la nuit se fait plus pesante pour les hommes lorsque leur corps doit prendre obligatoirement du repos. Pour que le dormeur récupère ses forces la nuit, il doit vaincre ses appréhensions, conscient que son corps est à ce moment le plus vulnérable.

En nous intéressant aussi à d'autres faits divers criminels ${ }^{7}$, nous verrons qu'ils sont une source très riche dans la connaissance des pratiques sociales d'un groupe. Les images du quotidien que l'on perçoit dans les faits divers montrent que les sociétés n'ont pas fait que subir la nuit, elles

4. L'espace étudié dans cette étude comprendra le monde rural, les villes petites et moyennes qui n'ont pas encore un éclairage nocturne régulier et répandu. Ce dernier facteur change en effet les habitudes et les perceptions nocturnes.

5. Delumeau, Jean, La peur en Occident, paris, Librairie Arthème Fayard, 1978; ManNonI, Pierre, La peur, Paris, Presses Universitaires de France, 1982.

6. Le sommeil comme sujet d'étude historique a été seulement effleuré par les historiens du corps. On peut citer MARTIN, Philippe, "Corps en repos ou corps en danger? Le sommeil dans les livres de piété dans la seconde moitié du XVIII ${ }^{e}$ siècle ", Revue d'histoire et de philosophie religieuses, 2000, Tome $80 n^{\circ}$ 2, p. 247-262; MILHOU-Roudie, Anne, «Le sommeil réprimé et le sommeil réhabilité » dans REDONDO, Augustin (dir.), Le corps dans la société espagnole des $X \mathrm{VI}^{\mathrm{e}}$ et $\mathrm{XVII} \mathrm{e}^{\mathrm{e}}$ siècles, Paris, Publications de la Sorbonne, 1990; VERDON, Jean, «Dormir au Moyen Âge » dans la Revue belge de philologie et d'histoire. Sur les lacunes historiographiques de la question du sommeil voir GARNIER, Guillaume, Le sommeil sous l'Ancien Régime, mémoire de DEA, Poitiers, 2003, p. 40.

7. On s'intéressera dans cette étude principalement aux faits divers de viols, d'inceste, de meurtre ou de vols car ils sont plus riches d'informations concernant le rapport au corps violenté, aux peurs, aux pratiques quotidiennes ou bien encore à la mise en place de moyens de protection des biens et des personnes. 
ont tenté de protéger leur sommeil et ont construit, volontairement ou non, des rituels du coucher à cet effet. Regarder sous son lit, cacher une arme ou son argent sous son oreiller, se calfeutrer dans sa chambre, être à l'écoute du moindre bruit, sont des pratiques courantes. Si ce constat a déjà été avancé par certains historiens, il n'a jamais fait l'objet d'études poussées. II est donc important de voir dans quelle mesure les populations pouvaient vraiment se protéger et donc quelles étaient réellement leurs habitudes dans ce domaine, tout en se demandant si ces précautions étaient vraiment efficaces.

Plus on avancera dans cette étude, plus le monde du sommeil nous paraîtra un sujet foisonnant. Car les faits divers nous mettront aussi sur une autre piste de travail, étudiée seulement par un historien américain Roger Ekirch ${ }^{8}$. Selon lui, les hommes et les femmes des sociétés préindustrielles connaissaient un sommeil segmenté, à savoir quelques heures de sommeil (trois ou quatre) interrompues par une période de veille, avant d'avoir à nouveau un temps de sommeil quasiment identique au premier. Il y aurait donc dans une même nuit un "premier sommeil » et un « second sommeil ». On retrouve dans de nombreux témoignages cette référence à un «premier sommeil ». Cette thèse s'avérerait très intéressante si elle se vérifiait, car elle permettrait d'éclairer certains mouvements et déplacements nocturnes, voire d'expliquer la présence de témoins éveillés lors de certaines affaires.

\section{Le corps endormi face à ses peurs}

Dans son ouvrage sur les nuits parisiennes, Simone Delattre a parfaitement mis en lumière cet apprentissage du noctambulisme qui démarre à la fin du $\mathrm{xVIII}^{\mathrm{e}}$ siècle ${ }^{9}$. La ville de Paris s'est mise à apprivoiser la nuit pour sortir de la dichotomie habituelle jour/nuit. Sans entrer dans les détails de cette transformation, il faut tout de même remarquer que si les activités nocturnes ont quelque peu changé dans la capitale, elles restent fortement conservatrices en province. Alfred Delveau déclamait en 1866 dans Les Heures parisiennes : "Il y a des villes qui dorment d'honnêtes bourgeoises, pendant tout le temps consacré au sommeil - c'est-à-dire pendant la nuit ${ }^{10}$. » Ici, l'auteur a tout à fait raison de remarquer que dans les villes moyennes (mais aussi les villages et les bourgs) du premier XIX ${ }^{\mathrm{e}}$ siècle, on perpétue la tradition de la nuit réservée au sommeil et de la nuit non éclairée, favorable au libertinage et à la criminalité ${ }^{11}$. Le temps nocturne demeurait un temps difficilement maîtrisable, ce qui permettait de dissimuler certaines

8. EKIRCH, Roger, "Sleep We Have Lost : Pre-industrial Slumber in the British Isles", American Historical Review, Avril 2001.

9. DelatTRE, Simone, Les douze heures noires. La nuit à Paris au xIXe siècle, Paris, Albin Michel, 2000.

10. Ibidem, p. 25.

11. Pour une présentation complète de l'«Ancien Régime» nocturne se reporter à DelATTRE, Simone, Les douze heures noires..., op. cit., p. 22. 
aventures, heureuses ou malheureuses ${ }^{12}$. Les faits divers se faisaient alors l'écho de certains de ces épisodes. Joseph Berthelot, même s'il n'a pas prémédité son crime, a tout de même profité de la nuit pour le camoufler. Aussi étonnant que cela puisse paraître, l'assassinat s'est effectué sans le moindre bruit. De colère Joseph Berthelot a frappé sa femme d'un coup de pierre sur la tête et comme cette dernière était endormie, elle n'a poussé aucun cri. Et encouragé par le fait que son acte n'avait alerté personne, il décide, cette fois volontairement, de prendre toutes les précautions possibles pour traverser la cour commune et atteindre discrètement le puits. Charles Martin Boutin, charron demeurant à Méré et plus précisément à proximité du puits, a déclaré sous serment n'avoir cette nuit-là « rien vu et rien entendu ${ }^{13} »$. Le criminel a utilisé l'obscurité de la nuit. Ce lien entre la nuit et la criminalité, de nombreux historiens l'ont remarqué ${ }^{14}$. Pourtant, le rapport très étroit qu'entretiennent la nuit et le sommeil, et donc par la suite le sommeil et la criminalité, n'a jamais été abordé. En rentrant plus dans le détail, on s'aperçoit que le premier complice du fait divers étudié dans cet article n'est autre que le sommeil. Anne Berthelot dormait lorsque son mari l'a frappée, et c'est en partie pour cela qu'elle n'a pas crié. Le sommeil a rendu cette femme encore plus vulnérable au crime. Mais les hommes du $X x^{e}$ et des siècles précédents avaient-ils pour autant conscience de cette dangerosité du sommeil ? On ne peut pas le nier au regard des pratiques et des représentations du sommeil de ces sociétés. Même si le sommeil est incontestablement un bienfait pour tous, le repos nécessaire d'une journée de labeur et l'élément qui rassemble pauvres et riches, il est aussi considéré dans les proverbes comme "la mort de la vie de chaque jour ${ }^{15}$ » ou bien encore "le frère de la mort». Communément, cette métaphore a pour sens la ressemblance physique entre un corps endormi et un corps mort. Ne dit-on d'ailleurs pas que la mort est un repos éternel? On peut pourtant y déceler un autre sens. Du sommeil à la mort il n'y a pas qu'une ressemblance, il n'y a aussi qu'un pas. Beaucoup ont connu la peur de s'endormir un soir pour ne pas se réveiller le lendemain. Les histoires d'hommes enterrés vivants ne manquent pas et elles se propagent encore au début du $\mathrm{XIX}^{\mathrm{e}}$ siècle ${ }^{16}$. II est difficile pour des populations faiblement instruites de comprendre comment elles peuvent perdre connaissance chaque

12. La ville de Poitiers, par exemple, a pourtant mis en place un éclairage public à l'huile en 1804. Mais les habitants ne manquent pas de rappeler tout au long du siècle, à propos de cet éclairage, la faible luminosité des réverbères qui ne leur permet d'évoluer que difficilement dans la ville en temps d'obscurité.

13. Arch. dép. de la Vienne, 2 U 542.

14. On peut penser à Benoît Garnot, Robert Muchembled, Arlette Farge ou bien encore Simone DelatTRE.

15. William Shakespeare dans Macbeth.

16. C'est seulement dans la première moitié du XVIII ${ }^{\mathrm{e}}$ siècle, que les médecins se sont emparés de la question pour dénoncer l'un des graves dangers de l'époque : les sépultures hâtives. On proposa alors, à la fin du xvIII ${ }^{\mathrm{e}}$ siècle, l'institution de "lieux de dépôts ", où les corps resteraient sous surveillance jusqu'au début de la putréfaction, afin qu'on puisse être absolument sûr du décès. Le progrès ne fut pas réalisé en France, mais en 
jour et se réveiller ou bien pourquoi, justement un jour, sans symptôme apparent, elles ne peuvent pas se réveiller.

Dormir suppose alors d'oublier ces considérations, ce que bon nombre réussissaient à faire, éreintés par les journées de labeur. Concrètement, les légendes ou les histoires fantasmées n'étaient pas omniprésentes comme pouvaient l'être les craintes envers "l'autre", celui que l'on ne connaît pas et qui nous touche pendant le sommeil. La personne qui va dormir s'expose bien plus encore qu'à d'autres moments de la journée à être vulnérable, soit au crime, soit au vol. Cette notion de vulnérabilité impose aux dormeurs un certain nombre de rituels pour se protéger. En dehors de quelques grandes villes comme Paris, la surveillance nocturne varie, allant souvent d'une totale inefficacité (faute de moyens et de personnel) à une quasi-inexistence, comme c'est le cas dans de nombreuses campagnes. Les seules mesures régulières mises en place par les autorités se résumaient simplement à inciter les populations à dormir uniquement de nuit ${ }^{17}$, quitte à leur faire peur :

"La nature a voulu que la nuit soit le temps du sommeil. Rien de plus contraire à la santé que de veiller la nuit. C'est le plus grand des malheurs, qu'un usage aussi destructeur de la santé soit si fort à la mode. Nous voyons tous les jours combien le défaut de sommeil dans le temps convenable ruine promptement le tempérament le mieux constitué de ceux qui font du jour la nuit et la nuit le jour ${ }^{18}$.»

Les deux objectifs de cette prévention - soutenus par les médecins portaient bien évidemment sur la volonté de l'Église d'utiliser la nuit pour faire affirmer leur piété aux fidèles (par la prière et le sommeil) et sur la résolution des possédants de sauvegarder leur patrimoine ${ }^{19}$. Quoi qu'il en soit, on ne peut que constater qu'une grande majorité des hommes du $x x^{e}$ siècle allaient se coucher en sachant qu'une minorité d'entre eux bravaient l'ordre public pour déambuler dans les rues. Cette persistance de "coureurs de nuit » dans la société française au long des siècles alimente l'idée que chacun peut passer un jour de l'état de dormeur à celui de victime. Sans occuper quotidiennement l'esprit de nos dormeurs, ces appréhensions ressurgissaient lors de certains événements : maladies, rumeurs, guerres, crimes ou vols à proximité de chez soi. C'est le cas de François Honoré Caury qui, une nuit de mars 1817, se retrouve confronté

Allemagne. Ces premières funeral homes étaient appelées vitae dubiae azilia, ou ce qui est moins joli, obituaires.

17. Il s'agit d'une mesure qui n'est plus applicable après la première moitié du XIXe siècle puisqu'en France la Révolution industrielle va développer des horaires différents, notamment du travail de nuit qui jusque-là n'était réservé qu'à certaines professions bien définies (boulanger par exemple).

18. Buchan, Guillaume, Médecine domestique, ou traité complet des moyens de se conserver en santé, de guérir $\&$ de prévenir les maladies, par le régime $\&$ les remèdes simples, Paris, chez Froullé ( $3^{e}$ édition), 1783, tome 1, p. 241.

19. Les médecins rejoignent d'ailleurs l'Église en interdisant le sommeil diurne. Seule la « méridienne », sieste de quelques minutes, est tolérée dans les pays du sud. 
à ses propres peurs. II déclare que cette nuit " une sorte de saisissement s'empara de [lui] ${ }^{20}$ " lorsqu'il s'aperçut que deux hommes étaient en train de forcer sa boutique. Même si François Honoré Caury se trouvait à une bonne distance des voleurs, il fut pris d'une angoisse extrême qui l'immobilisa. À ce moment-là, c'est le rapprochement entre ce qu'il avait sous les yeux et les histoires qu'il avait entendues, qui a dû lui faire ressentir une telle crainte. Cette angoisse devait être réellement d'une forte intensité car la victime déclare, assez fière d'elle, avoir eu malgré ce saisissement, le courage et la force de crier aux voleurs. On retrouve fréquemment ce genre de dépositions où les témoins et les victimes sont submergés par leur peur. Ils se replient alors dans une inertie qui réduit leur possibilité de réagir. Une servante, interrogée sur la tentative de vol survenue chez ses maîtres le 27 décembre 1762 sur les 11 heures du soir, témoigne de cette aversion. Elle déclare,

«avoir été réveillée par les violentes secousses par la grille de fer [...] que les frayeurs qu'elle a ressenties de tous ces mouvements lui a fait grande impression, qu'elle n'a pas osé remuer de son lit, ni su crier soit à cause de la peur dont elle a été saisie, soit à cause du gros rhume qu'elle $a$, qu'après avoir resté dans cet état d'affliction et entendant toujours que l'on secouait la grille avec force elle a toussé et s'est fait entendre ${ }^{21} »$.

Si Anne Berthelot n'a pas connu au moment de son assassinat cet effroi (qui d'ailleurs aurait pu la sauver), elle fut victime de l'obscurité nocturne et de son assoupissement. Comme chaque soir, elle est allée se coucher et comme souvent elle était ivre. Après avoir injurié plusieurs fois son mari, elle s'endort sans avoir conscience d'un réel danger. La dispute avec son mari aurait pu s'arrêter là. Elle ne soupçonnait vraisemblablement pas que, contrairement aux autres fois, son mari n'allait pas supporter l'affront ${ }^{22}$. Même si Joseph Berthelot n'a pas détaillé les raisons de son " coup de sang ", on peut essayer de comprendre quelles étaient les raisons qui l'ont poussé après de nombreuses années de mauvais ménage, à l'assassiner. Il témoigne que cette nuit-là, lassé des injures, il en était arrivé à devoir se coucher sur sa maie. Un lieu tellement inconfortable pour dormir, que quelques minutes après s'y être installé, notre criminel toujours furieux et fatigué de la situation (et de sa journée) s'empare d'une pierre pour frapper sa femme. Un corps manquant de sommeil a agressé un corps endormi. Et pas n'importe quel agresseur : le mari même de la victime. Ce genre d'affaire ne peut que renforcer l'idée que la nuit est un moment où tout peut arriver. Il est donc impossible de dormir, tout en étant concentré sur le monde extérieur. Doit-on pour autant dire que le corps endormi ne peut inexorablement pas échapper aux dangers de la nuit? Est-il voué à toujours jouer le rôle de victime ou bien peut-il se protéger?

20. Arch. dép. de la Vienne, 2 U 540.

21. Arch. dép. de la Vienne, G 668.

22. Beaucoup de témoins interrogés ont déclaré qu'il était fréquent qu'Anne Berthelot injurie son mari, sans qu'ils en arrivent aux mains. 
Le corps endormi « sur la défensive »

Un premier élément de réponse se trouve dans l'étude du paysage sonore nocturne. À la suite de Lucien Febvre, Jean Pierre Gutton a constaté que nos ancêtres savaient parfaitement interpréter les bruits du dehors ${ }^{23}$. La nuit, les témoins ne voyaient pas mais entendaient parfaitement. François Honoré Caury, dont nous avons parlé précédemment, a déclaré qu'étant couché dans sa chambre qui donne sur la rue, il fut éveillé la nuit du 8 au 9 mars 1817 vers une heure par un bruit dans la rue. Heureusement pour lui, car les deux hommes d'une assez grande taille, vêtus l'un et l'autre de grandes redingotes qu'il aperçoit, étaient en train de forcer l'entrée de sa boutique ${ }^{24}$. II n'y a pas que les bruits suspects qui peuvent interpeller les dormeurs. Pierre Colasson, laboureur demeurant à Vivonne dans la Vienne, est témoin de cette même affaire de vol. Il se souvient avoir entendu une heure après minuit, une voiture passer devant sa porte. II a été éveillé de son sommeil par un bruit assez banal, dont il ignorait à ce moment-là l'importance. Un dernier exemple encore plus frappant du crédit accordé au son par la société du premier $\mathrm{XIX}{ }^{\mathrm{e}}$ siècle nous est offert par une affaire d'infanticide de 1823. Ce crime est découvert par Pierre Patreau, demeurant à Verrières, qui vers trois heures du matin a entendu ses voisins dissimuler dans leur jardin le corps d'un nouveau-né ${ }^{25}$. Quelques bruits de pelle et des paroles entendues ont permis à Pierre Patreau de certifier aux autorités que ses voisins étaient bien coupables d'un infanticide. Dernier détail à noter, Pierre Patreau est aveugle et à aucun moment son témoignage ne fut mis en cause.

Incontestablement, les hommes des sociétés traditionnelles prêtaient une oreille plus qu'attentive aux bruits et aux odeurs. Mais il faut aussi remarquer qu'il leur était plus facile de percevoir des sons puisque l'environnement sonore nocturne de cette époque était assez calme. Rien d'étonnant à cela quand on sait ce qui attendait les travailleurs chaque matin. Les bruits suspects comme les charivaris, la circulation de chevaux, les bagarres ou les déambulations d'ivrognes, étaient très rapidement repérés et dénoncés dès le lendemain matin auprès des autorités. Les municipalités rappelaient fréquemment à certains métiers qu'ils ne devaient pas travailler aux ouvrages capables de troubler le repos et la tranquillité des malades et des gens de cabinet avant 6 heures ou 7 heures du matin ${ }^{26}$. II faudra attendre les débuts de la Révolution Industrielle pour que le bruit des machines vienne bouleverser les habitudes nocturnes.

Le silence nocturne rend donc suspect tout bruit. II est difficile pour les criminels d'évoluer dans un univers obscur (I'usage de lumière pourrait donner l'alerte) sans produire des sons qui éveilleraient les victimes ou les voisins : porte fracturée, franchissements de barrières... À cela, il faut ajou-

23. GuTTON, Jean Pierre, Bruits et sons dans notre histoire, Paris, PUF, 2000, p. 73.

24. Arch. dép. de la Vienne, 2 U 540.

25. Arch. dép. de la Vienne, 2 U 547.

26. Ibidem, p. 84. 
ter que la promiscuité des habitations, si souvent décriée quand on parle d'intimité, offre aussi une protection aux dormeurs. La faible épaisseur des murs permet bien souvent aux voisins d'être alertés en cas de danger, ces derniers n'hésitant pas à intervenir ou à donner l'alerte. Dans le cas d'Anne Berthelot, l'agresseur était son mari et il n'eut donc aucun effort particulier à faire pour s'approcher sans bruit de sa victime. Un dernier facteur plus difficilement perceptible reste à étudier. II touche directement à la physiologie du corps endormi. Le silence nocturne jusqu'au XIX ${ }^{e}$ siècle a sans doute conditionné une qualité de sommeil qui ne sera transformée, d'abord dans les villes, là aussi, qu'avec la Révolution industrielle. Les traités de médecine le signalent, il est plus facile de s'endormir lorsque l'on n'est entouré d'aucun bruit. De la même manière, les dormeurs du passé qui évoluaient dans cet univers avaient, semble-t-il, un sommeil moins profond que les dormeurs des sociétés industrialisées, (puis des sociétés de consommation) qui devront s'habituer à de nouveaux éléments sonores. Les populations comptaient indéniablement sur le fait que leur sommeil ne pouvait pas être lourd au point de ne pas entendre le bruit de fracture d'une porte, les voleurs sachant crocheter une porte dans le silence n'étant pas si nombreux. Ce sont donc les dangers nocturnes et le manque de surveillance efficace qui ont entraîné des générations " de dormeurs aux aguets ».

Et si les dormeurs comptaient sur la possibilité de réagir rapidement en cas de réveil inopiné, ce n'est pas seulement dans le but de se protéger eux, mais aussi de protéger leurs biens. Car il est un rituel du coucher qui, s'il n'est pas inconnu des voleurs, est le seul, aux yeux des dormeurs, qui protège leur patrimoine. Le meilleur moyen de le préserver, c'est de l'avoir à proximité de soi quand on dort. La chambre se fait le lieu d'accueil de migrations surprenantes. Louis Siballeau, voyageant avec son domestique, s'arrête une nuit à l'hôtel de l'Épée à Saumur. Transportant avec lui son argenterie, il l'enferme à clef dans l'armoire de sa chambre, puis laisse la clef sur la table de nuit qui se trouve auprès de son lit. Malheureusement pour lui, le voleur s'est emparé de la clef et du contenu de l'armoire sans qu'il s'en aperçoive. D'autres entreposent leur argenterie dans une armoire située dans la pièce principale et gardent la clef dans leur chambre. C'est le cas de Madame Gilot, aubergiste à Saumur, qui a I'habitude de garder dans sa chambre la clef de son armoire située dans une autre pièce. Cela n'a pas empêché le voleur de s'introduire dans sa chambre pour prendre la clef laissée par mégarde sur la table de nuit, puis de s'emparer de l'argenterie.

Enfin, d'autres préfèrent cacher leurs précieux biens au plus près d'eux, à l'intérieur même de leur lit. À la fin de la Révolution on retrouva l'épée des rois de France dite Joyeuse, que l'on disait avoir appartenu à Charlemagne et qui avait disparu depuis 1793, tout simplement dans un garni parisien, sous le sommier d'un modeste lit ${ }^{27}$. Autre exemple, celui du juge Benoît

27. Audinot, Didier, Énigmes inexpliquées de I'histoire de France, Paris, Éditions Granger, 2005, p. 294. 
qui, lorsqu'il devait s'absenter un jour ou deux de Vouziers pour ses affaires, ne manquait pas de faire de chaudes recommandations avant de par$\operatorname{tir}^{28}$. Connaissant de par sa fonction la criminalité nocturne, il instruisit sa femme, son fils et leur domestique Louise Feucher, des précautions à prendre pour sauvegarder le patrimoine familial. La nuit du 8 au 9 novembre 1829, en l'absence de son mari, Madame Benoît le remplace et inspecte chaque fenêtre et chaque porte avant de mettre le précieux sac d'or renfermant six mille francs sous son oreiller. En cas de vol, il faudrait d'une part aux voleurs devoir fracturer une ouverture et d'une seconde part s'approcher au plus près de la propriétaire de la maison au risque de la réveiller. Si les dormeurs ne pouvaient pas assurer totalement leur sécurité, ils tentaient donc par ces différentes pratiques de décourager les éventuels voleurs.

Revenons pour terminer sur le fait divers concernant la famille Benoît, qui est encore riche d'enseignement. Nous avons laissé Madame Benoît en train de dissimuler l'or familial le soir du 8 novembre 1829 . Quelques instants après, leur voisin, le chirurgien Dossereau, est réveillé par " des hurlements hystériques » venant du fils de Madame Benoît. Concluant tout de suite à un cambriolage, il saute alors du lit pour aller chez ses voisins; lorsqu'il arrive dans la chambre de Madame Benoît, il la découvre gisant sous les couvertures. L'or avait bien évidemment disparu. À l'examen, la défunte paraissait avoir reçu la mort sans se défendre; pourtant une telle blessure n'avait pas pu la laisser sans réaction. Si les cris du fils ont tout de suite alerté le voisin, pourquoi ce dernier n'a-t-il pas entendu ceux de Madame Benoît? C'est le fils lui-même qui va nous apporter des éléments de réponse. Frédéric Benoît a indiqué qu'il s'était couché la veille vers huit heures et demie du soir, puis il avait été tiré de son sommeil par un cri de sa mère; il ne s'était pas inquiété, car celle-ci était sujette à ce genre d'exclamations lorsqu'elle faisait des cauchemars. Il ne s'était levé que lorsqu'il avait entendu d'autres bruits plus suspects. C'est une expression habituelle du corps endormi (un cauchemar) qui a jeté le trouble dans l'esprit du fils de la victime. Elle a crié, mais pas assez pour que le voisin en soit informé, et d'une manière trop proche de ses habitudes pour que son fils s'en inquiète. Les enquêteurs n'ont en aucun moment remis en cause ce témoignage. Le corps endormi ne réagit pas d'une manière rationnelle. On espère pouvoir se réveiller en sursaut de manière à alerter ses proches, mais on ne contrôle pas le cauchemar (appelé aussi incube) qui, si on écoute les médecins, provoquent les mêmes réactions qu'une agression ${ }^{29}$. De nombreux médecins ont tenté avec succès de décrire les manifestations des cauchemars. On peut retenir celle de Bond, certainement une des plus saisissantes sur la question :

28. Ce fait divers est raconté dans l'ouvrage de DraChLINE, Pierre, Le fait divers au XIXe siècle, Paris, Hermé, 1991, p. 117-124.

29. Voir à ce sujet JonEs, Ernest, Le cauchemar, Paris, Éditions Payot \& Rivages, 2002 [1 ${ }^{\text {re }}$ éd. 1931, chez Mrs Katherine Jones]. 
"Le cauchemar saisit généralement les personnes dormant sur le dos et il débute souvent avec des rêves effrayants auxquels succèdent rapidement une respiration difficile, une oppression violente sur la poitrine et une privation totale des mouvements volontaires. Dans leurs souffrances, ces personnes soupirent, grognent, émettent des sons indistincts et demeurent dans les mâchoires de la mort jusqu'à ce que, au moyen des efforts naturels les plus grands ou d'une aide extérieure, elles échappent à ce terrible état $d^{\prime}$ inertie ${ }^{30}$.»

Même si, comme nous allons le voir, le témoignage de Frédéric Benoît est faux, on peut accorder quand même un crédit à ce mensonge, puisqu'il décrit des pratiques jugées comme recevables par les autorités de l'époque. Ici, les descriptions des médecins rejoignent celles de Frédéric Benoît. Les cris de terreur auraient pu avoir pour origine un cauchemar. Mais si ce témoin essentiel a menti, où est donc la vérité ? Des dénonciations, des découvertes de preuves et des aveux vont alimenter ce fait divers avant que l'on découvre finalement le fin mot de I'histoire. Avec la complicité de la domestique Louise Feucher (qui à l'origine avait déclaré n'avoir rien entendu), Frédéric Benoît a assassiné sa mère pour s'emparer de l'or. Nous sommes dans le même cas qu'avec Anne Berthelot puisque le criminel avait l'avantage d'être déjà à l'intérieur de la maison de sa victime. On ne sait pas si cette dernière a crié, mais le criminel étant un proche, il a pu l'aborder sans problème et l'empêcher de crier d'une manière ou d'une autre. Par contre, il a pris soin de crier à son tour pour se faire entendre de son voisin. Il s'est par la suite disculpé en déclarant confondre des cris de cauchemar à des cris d'agression.

On peut donc constater que les rituels du coucher sont un objet d'enjeu pour les populations qui veulent se protéger, mais aussi de la part des criminels qui détournent le système ou bien qui s'en servent pour mentir et s'offrir un alibi. Le corps endormi évolue dans un univers qui prend en compte le bien-être du dormeur (silence, qualité de la literie...), les représentations du monde nocturne qui véhiculent certaines peurs, la volonté de protéger son corps et enfin un élément qui nous reste à voir, les rythmes du sommeil intégrés dans des contraintes naturelles (nuit, temps et saisons) et quotidiennes (le travail).

\section{Les rythmes du corps endormi}

En rencontrant des victimes endormies dans les affaires criminelles, on a de fortes chances de tomber sur des témoins eux aussi endormis. L'étude de leurs déclarations est très enrichissante dans la mesure où elle renseigne - en plus des détails de l'affaire criminelle - sur les pratiques sociales relatives au sommeil. À ce titre, on rencontre deux grands types de témoignages dans les faits divers nocturnes. Dans un premier temps, on peut trouver des témoins qui se sont réveillés ou qui n'étaient pas encore

30. Bond, J., An Essay on the Incubus, or Nightmare, 1753, p. 2. 
endormis au moment où ils sont devenus observateurs. Le 12 septembre 1699, Jean Cardin, citadin de Poitiers, se lève sur les 1 à 2 heures du matin pour "gaster de l'eau ${ }^{31}$ ». Il est alors à la fenêtre de sa chambre quand il aperçoit un homme déguisé avec un grand chapeau, entrer dans la maison du dit Boutin. Cette nuit-là, un vol de blé avait été commis et le Sieur Boutin, chez qui on retrouva du blé alors qu'il vivait dans la misère, fut accusé et condamné à cause de sa sortie nocturne. Le voleur s'était pourtant fait le plus discret possible, ce n'est qu'un concours de circonstance qui l'a condamné. Il avait choisi le meilleur moment, le milieu de la nuit quand tous ceux qui n'ont rien à se reprocher sont en train de dormir. Mais les faits divers ne sont pas tous peuplés de témoins aussi coopérateurs. Des personnes proches de certains événements s'aperçoivent qu'elles les ignorent à cause de leur sommeil. Dans certaines déclarations, on pourrait même percevoir les regrets de voisins qui n'ont rien entendu. Dans l'affaire concernant Anne Berthelot, au moins trois voisins ont déclaré n'avoir rien entendu le soir du crime, ce qui les étonnait puisqu'ils étaient à proximité du lieu du crime. Une voisine, Jeanne Verron, dont nous verrons attentivement le témoignage par la suite, pouvait entendre marcher chez la famille Berthelot. Alors pourquoi n'a-t-elle pas pu être réveillée par la querelle de ce couple?

Dans un deuxième temps, les faits divers nocturnes font apparaître qu'à des heures bien avancées de la nuit, certains témoins étaient bien éveillés et, pour une grande majorité d'entre eux, occupés loin de leur lit à diverses activités. Ce constat paraît bien étonnant face aux conceptions de l'époque qui poussaient encore, dans les campagnes du début du XIX siècle, à l'enfermement nocturne. Ces différents témoignages rendent compte de réalités plus complexes et diversifiées en ce qui concerne les pratiques liées au corps endormi : dormeurs profonds, dormeurs qui réagissent à une stimulation extérieure (cris, bruits...), personnes non endormies ou réveillées naturellement ou bien encore dormeurs troublés et malades ${ }^{32}$.

C'est tout d'abord le témoignage d'une dormeuse qui va nous informer sur la présence à cette époque de ces «non dormeurs». Voici les premières phrases de la déclaration de Jeanne Verron, proche voisine d'Anne Berthelot :

«La veille de la nuit où la femme Berthelot paraît avoir été tuée par son mari, elle avait, ainsi que son mari, passé la soirée avec nous dans la cour, lorsque je me suis retirée pour me coucher, ni le mari ni la femme ne l'étaient encore, et j'entendais même de chez moi la femme causer. II faut qu'il l'ait tuée dans le premier sommeil, car je n'ai rien entendu. D'assez bon matin,

31. Arch. dép. de la Vienne, G 652.

32. Cette dernière catégorie de «dormeurs troublés ou malades» doit apparaître dans cette étude, mais le sujet ne sera pas développé. II s'agit des corps endormis troublés par des facteurs extérieurs (non humains) comme les moustiques, punaises... et des personnes atteintes de maladies liées au sommeil comme l'insomnie, le somnambulisme, l'incontinence... 
j'ai bien entendu marcher dans sa chambre et je croyais que c'était elle qui se levait ${ }^{33}$.»

En allant se coucher, Jeanne Verron percevait encore les voix de ses voisins. À cette distance et encore éveillée, elle aurait dû entendre les cris supposés d'Anne Berthelot ${ }^{34}$. Selon elle «il faut qu'il l'ait tué dans le premier sommeil, car [elle] n'a rien entendu ». Elle utilise un terme très intéressant, sur lequel il convient de s'arrêter quelques instants. Par l'expression de "premier sommeil», cette femme entend les deux, trois voire quatre premières heures de sommeil. Ces premières heures de sommeil sont, aux yeux de ce témoin, particulièrement lourdes, puisqu'elles permettent de récupérer les forces de la journée. Scipion Dupleix le remarquait déjà au début du XVII siècle. À la question : "Pourquoi est-ce que le premier sommeil est plus fort \& plus profond \& qui tient les sens plus attachés \& plus assoupis? ", il répondait: "Pour ce qu'il y a lors plus grande quantité de vapeur au cerveau qu'après qu'on est reposé quelques temps \& que la digestion est faite ou à peu près achevée. Car la chaleur naturelle n'étant pas si occupée à l'intérieur commence à s'épandre \& consumer les vapeurs qui estoupaient les conduits des sens ${ }^{35}$. " C'est dans les premières heures du sommeil que le corps travaille le plus pour récupérer et donc affaiblit le plus le dormeur. On perçoit cette explication dans le témoignage de Jeanne Verron. Un bruit ordinairement assez fort pour être entendu (comme des cris) ne l'est pas forcément dans le premier sommeil. Ce premier constat ne semble pas avoir été discuté par les médecins et il était même partagé par un grand nombre de gens qui le savaient par leur propre expérience. Ils étaient tout aussi conscients qu'il n'était pas impossible de se réveiller, mais que c'était plus difficile. Jean Lunet cabaretier, demeurant à Loudun, accusé d'avoir tué sa femme, témoigne lui aussi de ce "premier sommeil »: "Le jeudi sur les huit à neuf heures nous nous couchâmes comme à l'ordinaire, je m'endormis de fait et dans mon premier sommeil je me réveillais en sursaut voyant la chandelle allumée ». Sauf qu'entre le moment où la chandelle a été allumée et le moment où Jean Lunet s'est réveillé, il s'est passé un laps de temps assez important pendant lequel sa femme a pu ouvrir les placards pour trouver de quoi boire.

Mais Roger Ekirch va plus loin dans l'interprétation de ce premier sommeil. Pour lui, il est constitutif d'un "sommeil segmenté ». S'appuyant sur des témoignages et sur des traités de médecine, Roger Ekirch tend à démontrer que le sommeil nocturne des sociétés d'Europe occidentale jusqu'à la Révolution industrielle, était composé de deux longs moments de sommeil et entre ceux-ci d'une période de veille pouvant aller jusqu'à

33. Arch. dép. de la Vienne, 2 U 542.

34. Ce que Jeanne Verron semble ignorer lors de sa déclaration, c'est que selon son mari Anne Berthelot n'a pas crié lorsque ce dernier l'a frappée. Quoi qu'il en soit, cela ne remet pas en cause la certitude de son explication du fait qu'elle n'ait rien entendu.

35. DuPLEIX, Scipion, La Curiosité naturelle rédigée en questions, selon l'ordre alphabétique, Paris, 1626. 
une heure ou plus ${ }^{36}$. Les deux phases de "premier et de second sommeil » avaient la même durée et les dormeurs se réveillaient généralement vers minuit. Bien entendu, tous les individus n'avaient pas exactement le même rythme de sommeil : certains ne se réveillaient après leur premier sommeil que pour finalement se rendormir, alors que d'autres, qui se couchaient aux alentours de minuit, ne se réveillaient généralement pas avant l'aube ${ }^{37}$. Ce "sommeil segmenté " aurait alors disparu avec la Révolution industrielle, qui a étendu la lumière artificielle à un plus grand nombre ${ }^{38}$. Le dernier facteur qui reste à prendre en compte c'est que ce sommeil segmenté ne semblait pas toucher les populations aisées qui s'étaient accoutumées d'un peu de lumière artificielle. Si l'on reprend les déclarations de nos acteurs de faits divers, nous avons uniquement eu à faire à des personnes plutôt modestes. Les faits divers semblent donc être une source confirmant l'existence d'un "sommeil segmenté ». L'expression de «premier sommeil » revient fréquemment et des témoins comme Pierre Patreau garantissent que des hommes connaissaient bien une période de veille utile pour surveiller les bêtes. On rencontre aussi cette période de veille chez les criminels (comme ceux dont nous avons parlé dans cette étude) qui se levaient en pleine nuit pour commettre les actes qu'ils ne pouvaient réaliser en plein jour : vol à l'étalage, vol dans les vergers, braconnage, infanticide...

Incontestablement, cette réflexion sur les rythmes du sommeil permet de relancer un débat parfois figé sur les pratiques du sommeil. II n'est plus question d'étudier séparément les dormeurs et les non-dormeurs. Au contraire de ce que l'on a pu penser depuis longtemps, ces deux groupes connaissaient bien les mêmes habitudes de sommeil. II faut alors rester prudent lorsque l'on parle d'individus « déviants » pour définir des personnes éveillées en plein milieu de la nuit. II faut le réserver aux seuls insomniaques ou noctambules qui font de la nuit le jour. II faut ajouter pour terminer qu'il reste tout de même certains points à clarifier pour pouvoir parler avec certitude de "sommeil segmenté ». Roger Ekirch utilise des sources très riches comme les livres de piété, l'iconographie, les traités de médecine... mais il aurait pu s'appuyer davantage sur les témoignages des gens ordinaires qui viennent confirmer la théorie des penseurs. Comme nous l'avons vu, les témoins interrogés dans les affaires criminelles du début du XIX ${ }^{\mathrm{e}}$ siècle font écho au «premier sommeil ». Qu'en est-il des siècles précédents? Un premier sondage dans le même type de sources entre 1700 et 1715 a permis

36. EKIRCH, Roger, At Day's close. Night in Times Past, W.W. Norton \& Company, New York, 2005, p. 300.

37. EkIRCH, Roger, "Sleep We Have Lost...," loc. cit. p. 365.

38. Roger Ekirch s'appuie sur les études menées en 1969 chez les Tiv au centre du Nigeria ou bien encore sur les expériences du Docteur T. Wehr qui s'est rendu compte que les sujets humains, privés de lumière artificielle quand il faisait nuit, et sur une période de plusieurs semaines, finissaient par afficher un rythme de sommeil tronqué identique à celui observé dans les foyers pré-industriels. Pour le détail des expériences, voir EKIRCH, Roger, ibidem, p. 367. 
de constater que cette expression n'était pas courante ${ }^{39}$. II est pourtant trop tôt pour en conclure quoi que ce soit. Pour faire la lumière sur les rythmes du sommeil, il faut continuer à être attentif aux moindres indices que peuvent nous apporter les témoignages en constituant un corpus plus étoffé et plus large.

Finalement, le fait divers est un bon indicateur de certaines pratiques du sommeil. L'auteur qui rapporte ce genre d'histoire, dans le souci de raconter les faits mais aussi d'intéresser le public, se fait l'écho de la quotidienneté. Et en étant attentif, on s'aperçoit que cette quotidienneté n'est pas forcément celle que l'on croyait voir. Ainsi, un terme assez simple, celui de "premier sommeil », ouvre de nouvelles perspectives sur la connaissance des rythmes du sommeil. Dès lors, on peut donc bien parler de « vie nocturne » quand on évoque les nuits et les dormeurs du xIx $x^{e}$ siècle, même s'il reste à définir clairement les rôles, les fonctions et les activités de chacun, lorsque les ténèbres recouvrent villes et campagnes.

Ainsi, le fait divers n'est pas un objet banal. Dans une certaine mesure, il fait réapparaître les peurs nocturnes de chacun. Sans non plus lui accorder une responsabilité qu'il n'a pas, on peut dire aussi qu'il rend (dans une certaine mesure) encore plus inquiétant le moment du sommeil, rappelant la vulnérabilité de chacun. Néanmoins, quelles que soient les préoccupations des futurs dormeurs, véhiculées ou non par les faits divers, elles disparaîtront en partie avec le sommeil. Car on dit de ce dernier avec raison, qu'il est surtout «l'oubli des peines ».

39. L'expression de "premier sommeil » n'a en effet été rencontrée que deux fois sur une soixantaine d'affaires. 
RÉSUMÉ

Le 11 octobre 1820, le petit bourg de Méré se retrouve au cœur d'un fait divers sanglant. Dans le puits de la communauté, le corps en état de décomposition d'Anne Berthelot est retrouvé. Le crime va être vite résolu : son mari Joseph Berthelot l'a assassinée une nuit de septembre, excédé par une nouvelle dispute. Banal ou étonnant, ce fait divers met aussi en avant un acteur peu mis à l'honneur dans l'historiographie récente: le corps endormi. En plein milieu de la nuit, Joseph Berthelot a frappé sa femme durant son sommeil, faisant ressurgir chez les plus proches voisins des sentiments anciens. La nuit mystérieuse est le domaine de tous les périls surtout pour le dormeur qui est à ce moment-là vulnérable. Le corps endormi est alors "sur la défensive » pour se protéger lui-même mais aussi pour protéger ses biens. Enfin, ce fait divers nous entraîne sur les rythmes de sommeil parfois mal connus des sociétés préindustrielles. Les dormeurs connaissaient vraisemblablement un "premier sommeil », une période de veille puis un "second sommeil », ce que Roger Ekirch définit comme un sommeil segmenté. Les dormeurs n'étaient donc pas prostrés chez eux mais pouvaient connaître des habitudes nocturnes licites (surveillance des bêtes, relation sexuelle, sommeil...) ou illicites (vols, rixes...).

\section{ABSTRACT}

On the $11^{\text {th }}$ October 1820, the little village of Méré becomes the heart of a bloody trivial event. Anne Berthelot's corpse is found in a state of decay in the well of the community. The crime will soon be solved: her husband, Joseph Berthelot, murdered her on a September night, exasperated with a new quarrel. Whatever trivial or amazing, this event enlightens a fact rarely honoured in recent historiography: the sleeping body. In the very middle of the night, Joseph Berthelot hit his wife while she was asleep, letting old feelings re-emerge in his next-door neighbours. A mysterious night is the world of all dangers, especially for the sleeper who is then vulnerable. A sleeping body is "on the defensive", so as to protect itself, but to protect its belongings too. In fact, this event carries us away on rhythms of sleep preindustrial societies didn't know very well. Sleepers probably experienced a "first sleep", a period of wake then a "second sleep", which Roger Ekirch defines as segmented sleep. So, sleepers were not prostrated at home but they could go through licit night habits such as looking after animals, having sexual intercourse, sleep or illicit habits such as theft or fights. 
